Supporting Information

\title{
Selective Promotion of Adhesion of Shewanella oneidensis on Mannose-Decorated Glycopolymer Surfaces
}

Thomas D. Young, ${ }^{\mathrm{a}, \mathrm{b}}$ Walter T. Liau, ${ }^{\mathrm{c}}$ Calvin K. Lee, ${ }^{\mathrm{c}}$ Michael Mellody, ${ }^{\mathrm{c}}$ Gerard C. L. Wong, ${ }^{\mathrm{a}, \mathrm{b}, \mathrm{c}}$ Andrea M. Kasko ${ }^{\text {b,c }}$ and Paul S. Weiss ${ }^{a, b, c, d}$

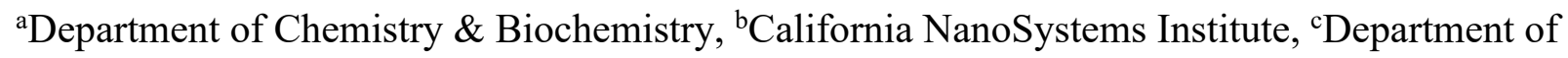

Bioengineering, ${ }^{\mathrm{d} D e p a r t m e n t}$ of Material Science and Engineering, University of California, Los Angeles, Los Angeles, California 90095, United States 


\section{CO-CULTURE COMPETITION EXPERIMENT}

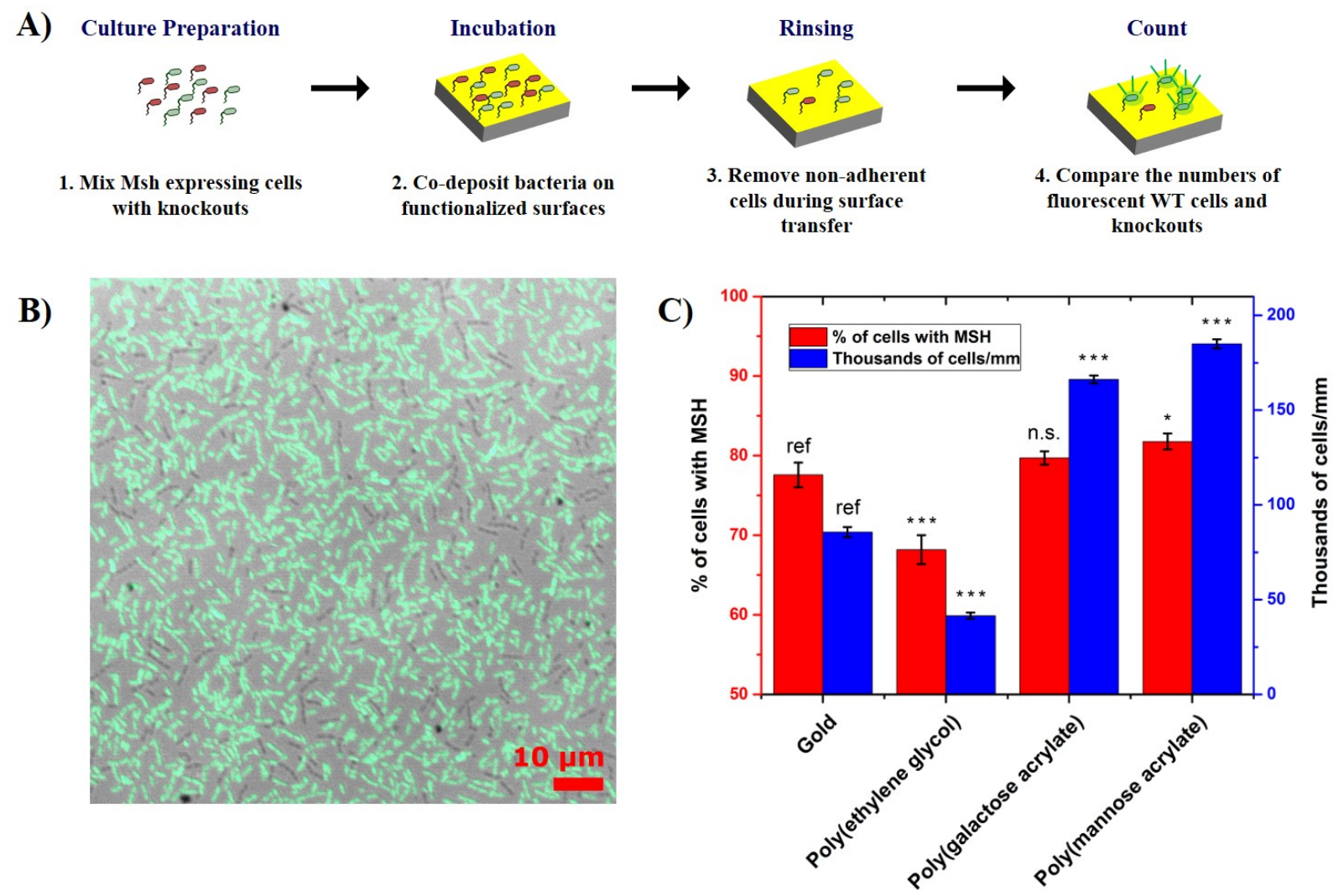

Figure S1. Surface colonization of competing $S$. oneidensis strains, containing $m s h A-D$ genes, and $\triangle m s h A-D$ knockout. (A) Method for determining the fraction of $m s h A-D$ expressing cells on the surface. The fraction of a strain is measured by counting the fluorescent strain (wild type) of cells versus the non-fluorescent $(\Delta m s h A-D)$ strain. (B) Example micrograph of the co-cultured surface. A fluorescence image is overlaid the brightfield image taken concurrently. The fluorescent strain is wild type, which produces mannose-sensitive hemagglutinin (MSH). (C) Fraction of fluorescent cells (red) and cell density (blue) on various surfaces. The $m s h A-D$ expressing strain is selectively enriched on polymannose surfaces compared to gold. Cell density is increased on the poly(saccharide acrylate) surfaces. Significance is marked by comparison to the bare gold reference. Error bars indicate standard errors of the mean. 
Selective enhancement of surface colonization by natively MSH possessing bacteria versus $\triangle m s h A-D$ genetic knockouts was possible on polymannose surfaces (Figure S1). Cultures of the two strains were mixed together with equal densities of the strains, then incubated on top of the various surfaces. The cultures were mixed and deposited simultaneously in an effort to standardize the concentration of signaling molecules secreted by the bacteria into the culture medium and to reduce the variability in lag time before colonization. The number of cells for each strain were counted on the various surfaces. The cell density of both strains increased on the glycopolymer surfaces, with polymannose increasing the most, and decreased on poly(ethylene glycol) surfaces versus bare Au. The cell density of wild-type cells was not proportional to the cell density of the $\triangle m s h A-D$ knockout cells. Polymannose surfaces showed the fraction of bacteria containing the $m s h A-D$ genes increase by $5.4 \pm 2.4 \%$ (SEM) versus bare gold surfaces.

Poly(ethylene glycol)-covered surfaces showed a reduction of the fraction of Msh expressing strain compared to bare gold surfaces. Poly(galactose) surfaces had a higher total cell density than gold (p-value $<0.001$ ), whereas in the results presented in Figure 3, the two sample types were not statistically distinguishable $(\mathrm{p}$-value $=0.087)$. This result may be due to extracellular GFP, which enters the culture medium when the fluorescent cells lyse, increasing total fluorescence. There may be more adsorbed extracellular GFP on the gold surfaces than samples with less nonspecific adhesiveness. The higher precision of the microscopy measurements can also explain why the difference was distinguishable. 
A)

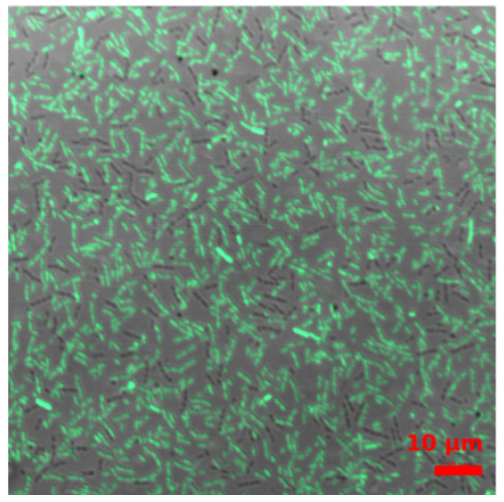

B)

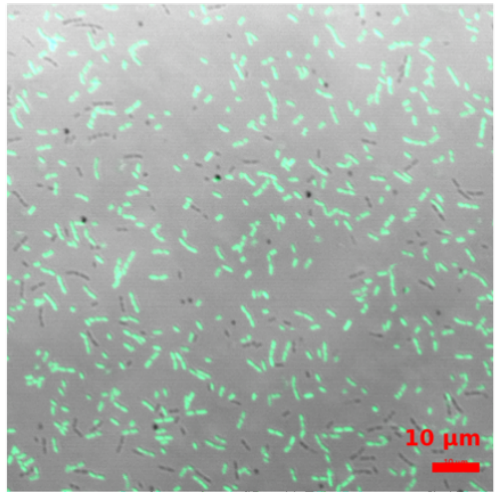

C)

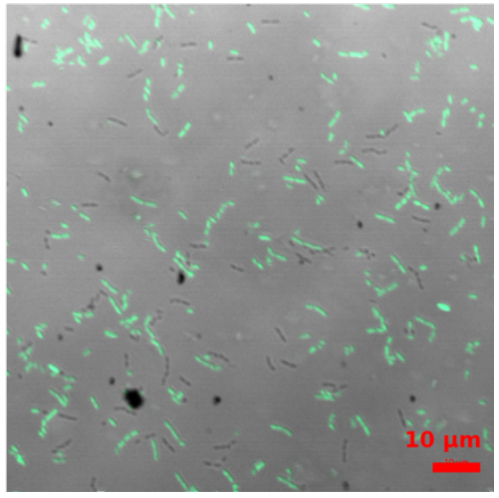

Figure S2. Representative micrographs of surfaces colonized by S. oneidensis wild-type (fluorescent) and $\triangle m s h A-D$ knockout (non-fluorescent) co-culture. (A) Polygalactose. (B) Bare gold. (C) Poly(ethylene glycol). Fluorescence images are overlaid with brightfield images that were taken concurrently.

\section{SUPPLEMENTAL CHARACTERIZATION}

\section{Ellipsometry}

Poly(mannose acrylate) surfaces were measured by ellipsometry to have an average dry height of $2.0 \pm 0.6 \mathrm{~nm}$ using a Gaertner LSE Stokes Ellipsometer (Gaertner Scientific Corporation, Skokie, Illinois, USA). Poly(galactose acrylate) surfaces were measured to have an average dry height of $1.2 \pm 0.9 \mathrm{~nm}$ with the same system. 


\section{Electrochemical Desorption}

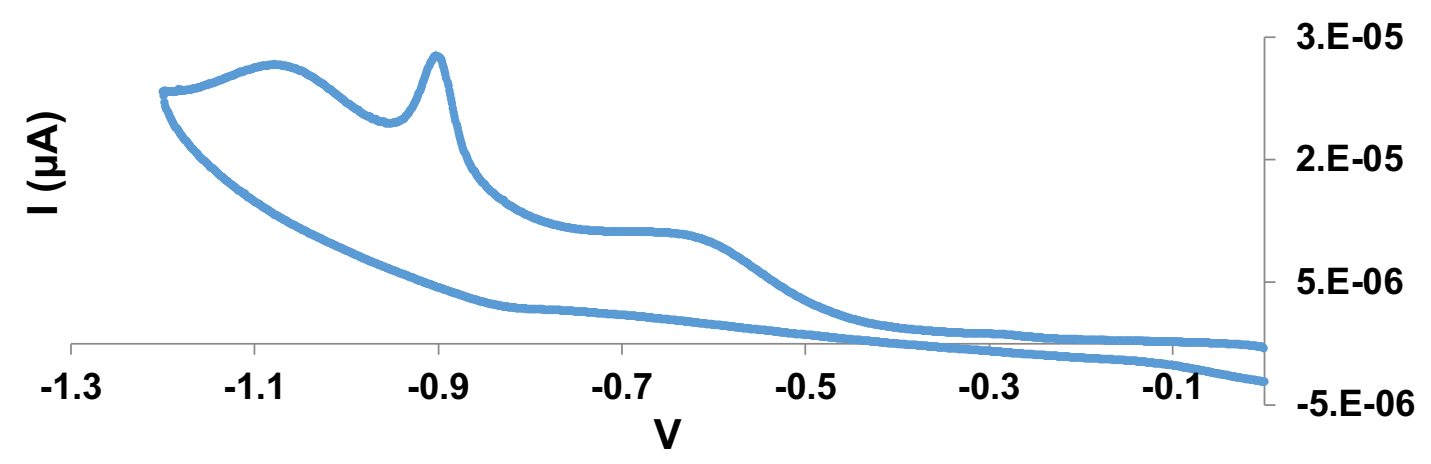

Figure S3. Electrochemical desorption of poly(2-O-( $\alpha$-D-mannosyl)hydroxyethyl acrylate) (polymannose) self-assembled monolayer. Anodic peak around $-0.9 \mathrm{~V}$ represents desorption of thiols from the surface. Integration of the anodic peak after subtracting a linear background reveals 6 electrons, corresponding to 6 thiols, per $\mathrm{nm}^{2}$ are transferred to the surface. The scan rate was $50 \mathrm{mV} / \mathrm{s}$.

\section{Contact angle (water)}

Polymannose (poly(2-O-( $\alpha$-D-mannosyl)hydroxyethyl acrylate)):

Advancing: $56.9^{\circ} \pm 0.6^{\circ}(\mathrm{SD})$

Receding: $36.2^{\circ} \pm 0.7^{\circ}(\mathrm{SD})$

Tethered mannose monomer (11-[(p-phenyl- $\alpha$-D-mannopyranosyl) aminocarbonyl methoxy hexa(ethoxy)]undec-1-yl-thiol):

Advancing: $38.9^{\circ} \pm 1.5^{\circ}(\mathrm{SD})$

Receding: $29.0^{\circ} \pm 2.0^{\circ}(\mathrm{SD})$ 


\section{Polarization modulation-infrared reflection-absorption spectroscopy}

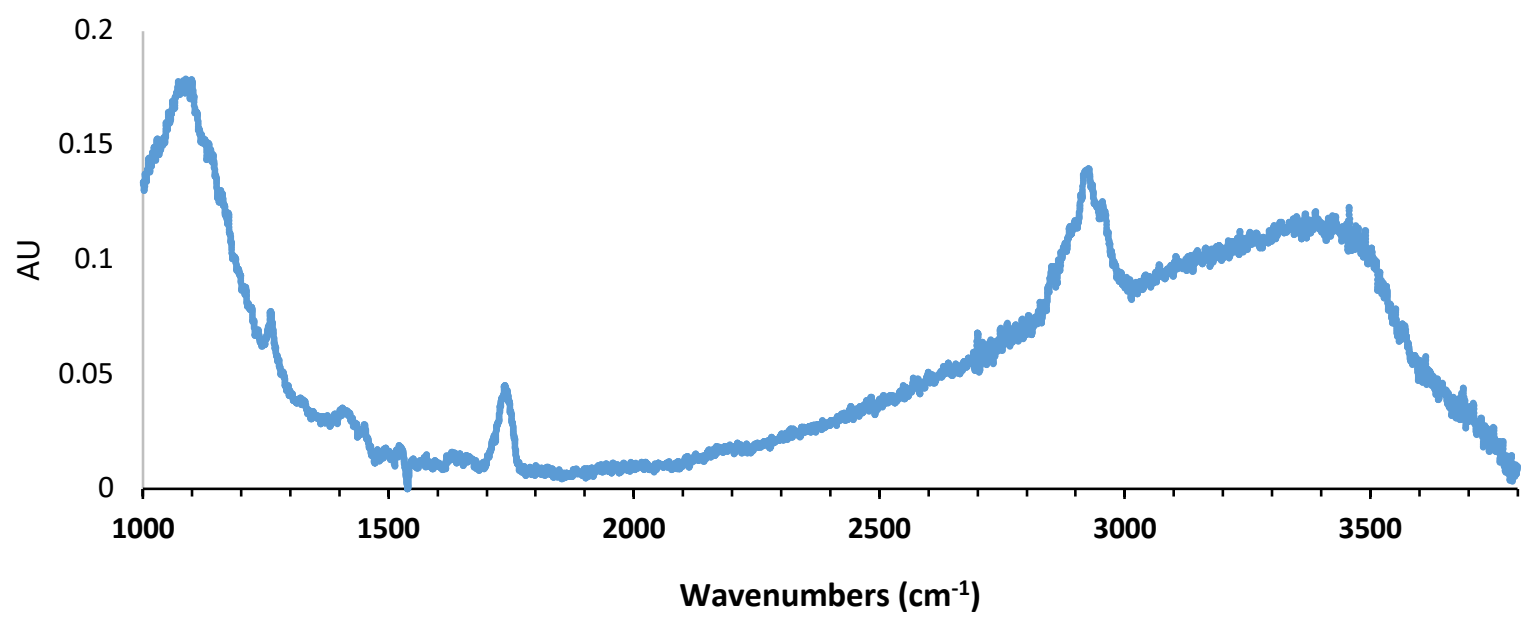

Figure S4. Polymannose (poly-2-O-( $\alpha$-D-mannosyl)hydroxyethyl acrylate) glycopolymer spectrum by polarization modulation-infrared reflection-absorption (PM-IRRAS). Several vibrational modes are present: ether stretching at $1100 \mathrm{~cm}^{-1}$, carbonyl stretching of the ester at $1750 \mathrm{~cm}^{-1}$, alkyl C-H stretch at $2900 \mathrm{~cm}^{-1}$, and a broad peak for alcohol stretching at $3400 \mathrm{~cm}^{-1}$. The spectral baseline has been removed. 


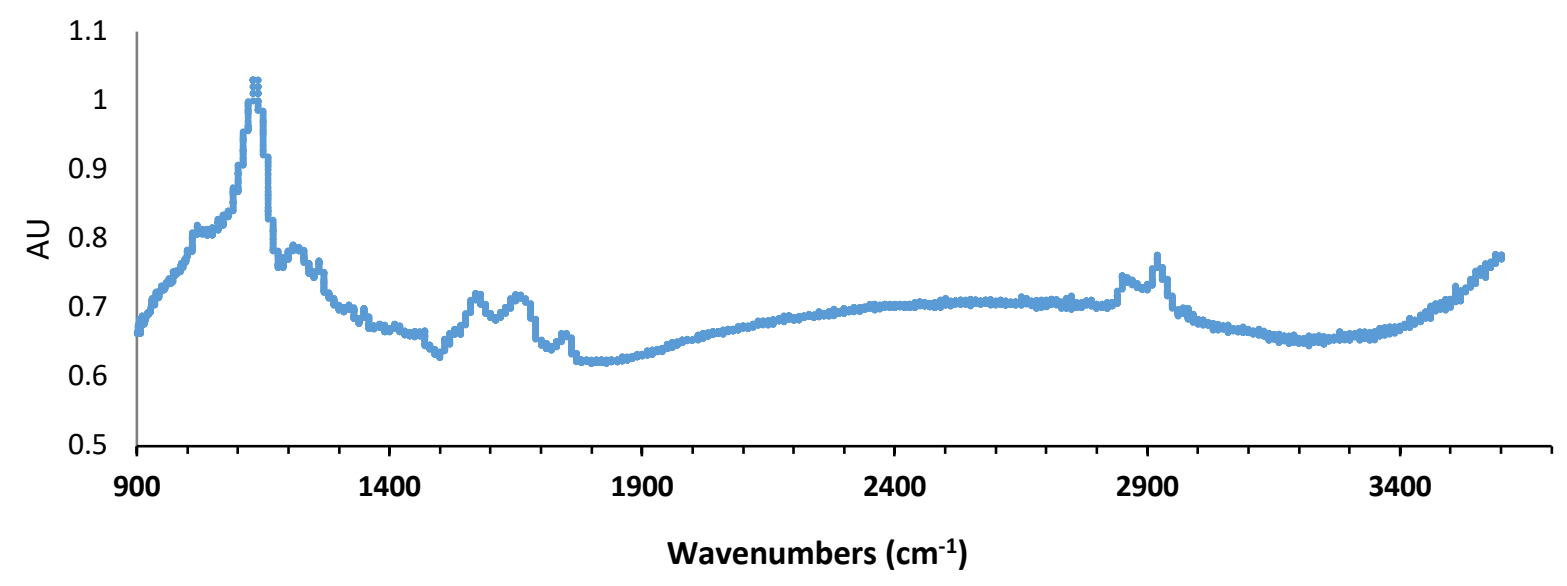

Figure S5. Tethered mannose monomer (11-[(p-phenyl- $\alpha$-D-mannopyranosyl) aminocarbonyl methoxy hexa(ethoxy)]undec-1-yl-thiol) PM-IRRAS spectrum. Ether stretching modes from the ethylene glycol units are visible at $1100 \mathrm{~cm}^{-1}$. Carbonyl of the carboxylic acid is mostly removed at $1750 \mathrm{~cm}^{-1}$ and replaced by the amide carbonyl stretch at $1650 \mathrm{~cm}^{-1}$ as the mannoside is added by amide bond formation. Alkyl and aromatic C-H stretching is present around $2900 \mathrm{~cm}^{-1}$. The spectral baseline has been removed. 
Nuclear magnetic resonance
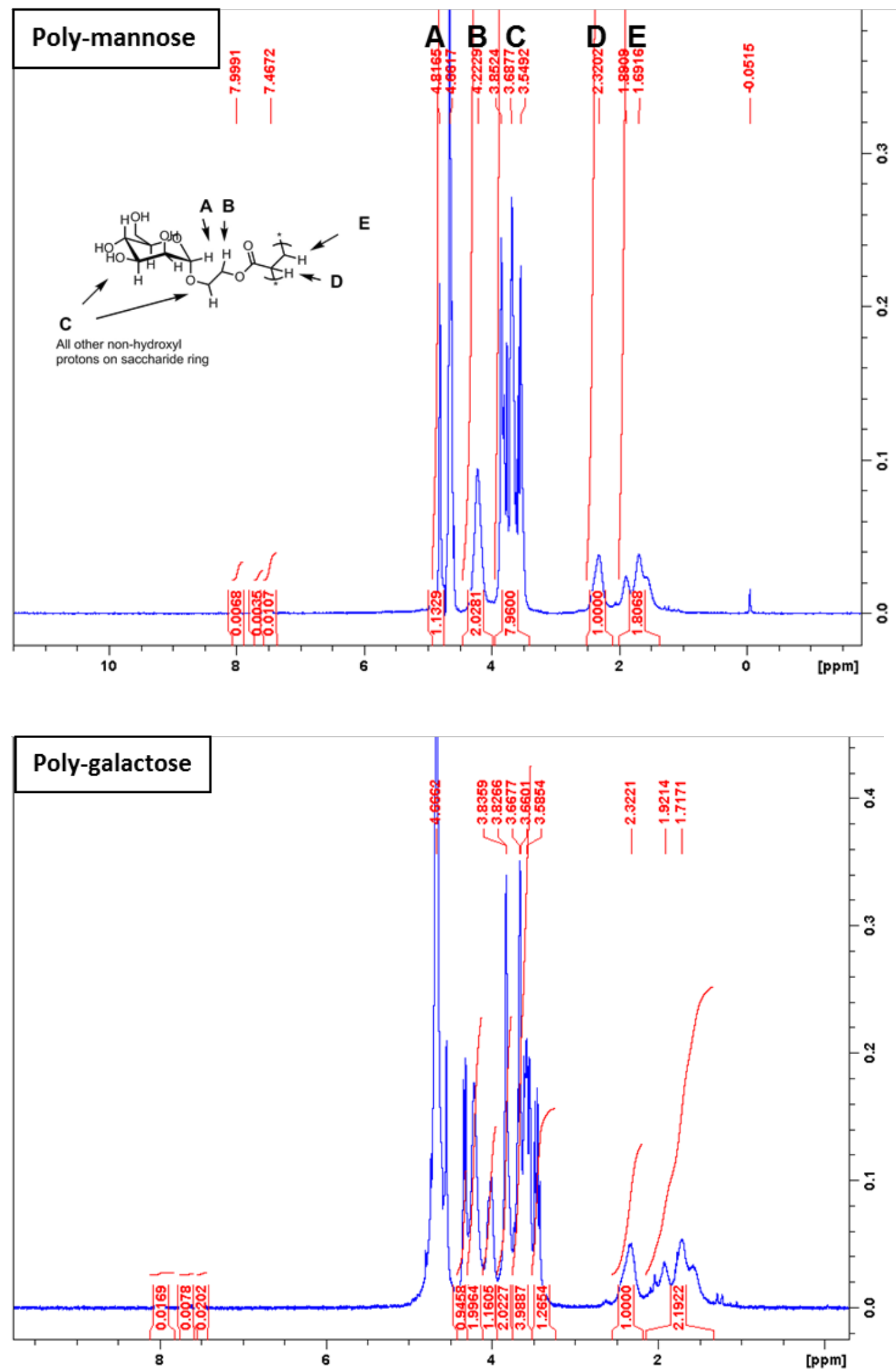

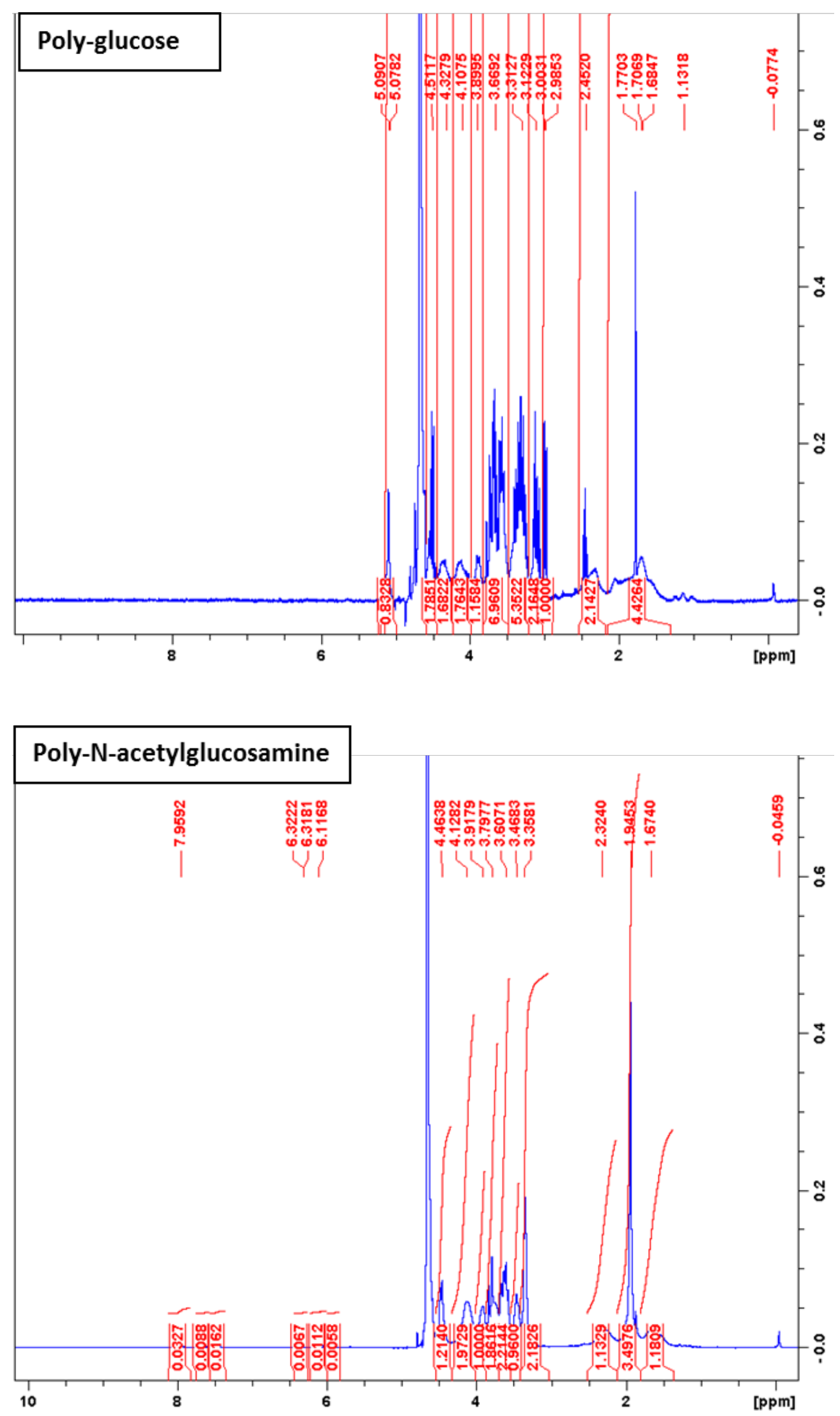
Figure S6. ${ }^{1} \mathrm{H}$ NMR spectra of the glycopolymers. The degree of polymerization can be determined by comparing the integral of aromatic protons, indicative of the chain terminus resulting from 4-cyano-4-(phenylcarbonothioylthio)pentanoic acid, to the relative proton signal of the glycopolymer. The amount of monomer conversion can also be determined by integrating the acrylate protons before purification by dialysis. Residual water peak observed at $4.8 \mathrm{ppm}$. 


\section{X-ray photoelectron spectroscopy}

A)

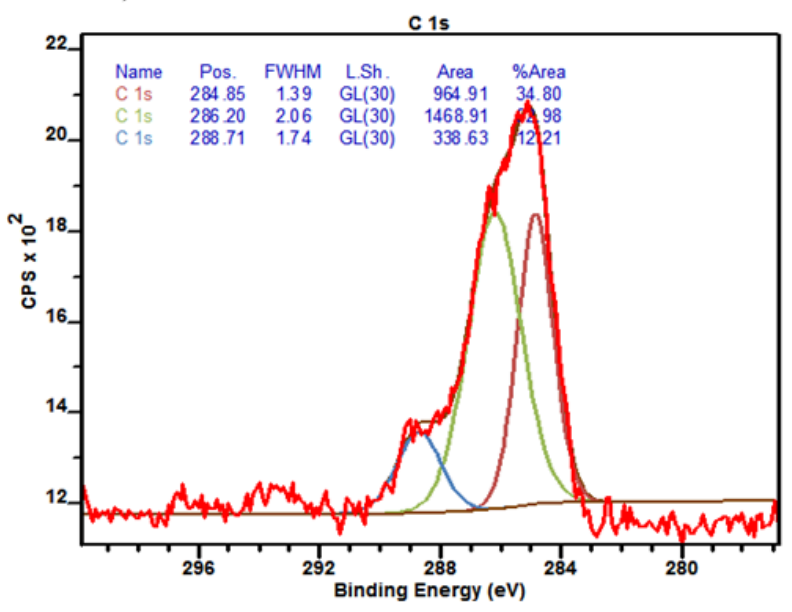

C)

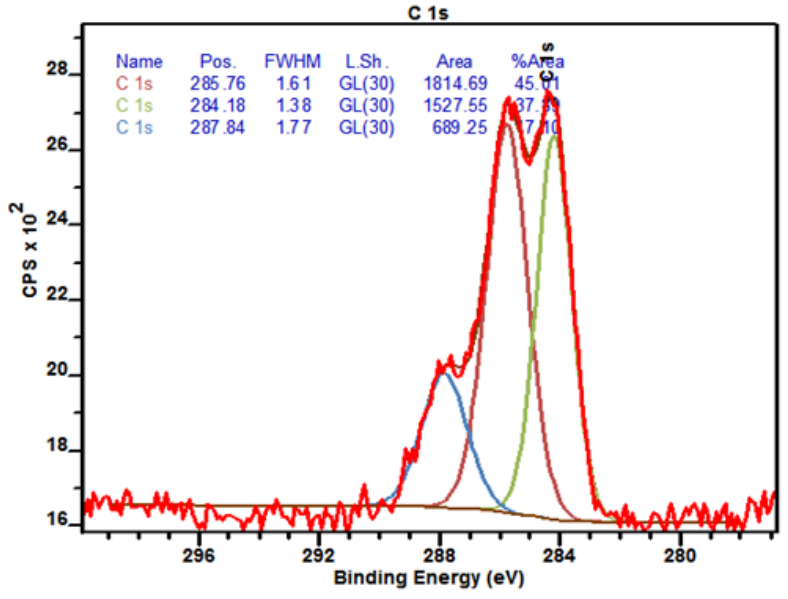

B)



D)

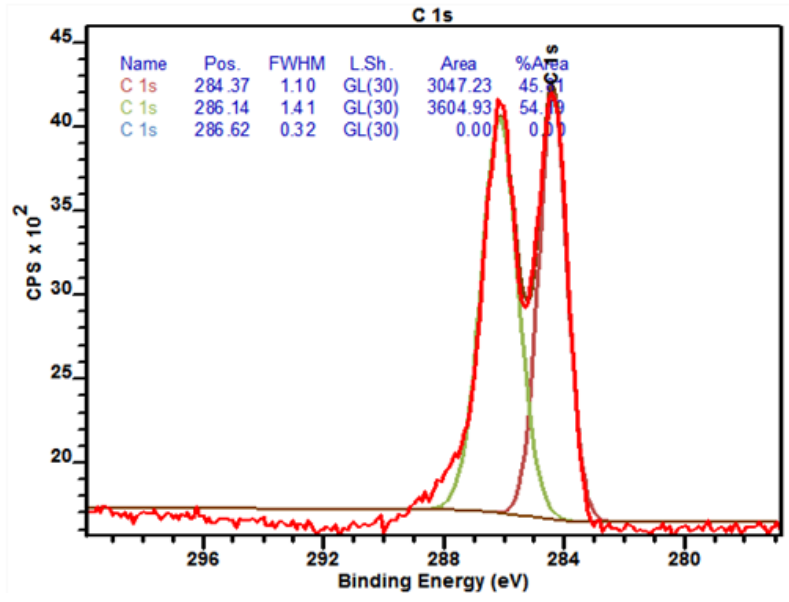

Figure S7. C 1s X-ray photoelectron spectra of A) polygalactose B) polyglucose C) poly $(N$ acetylglucosamine) D) tethered mannose monomer. 
A)



C)



B)

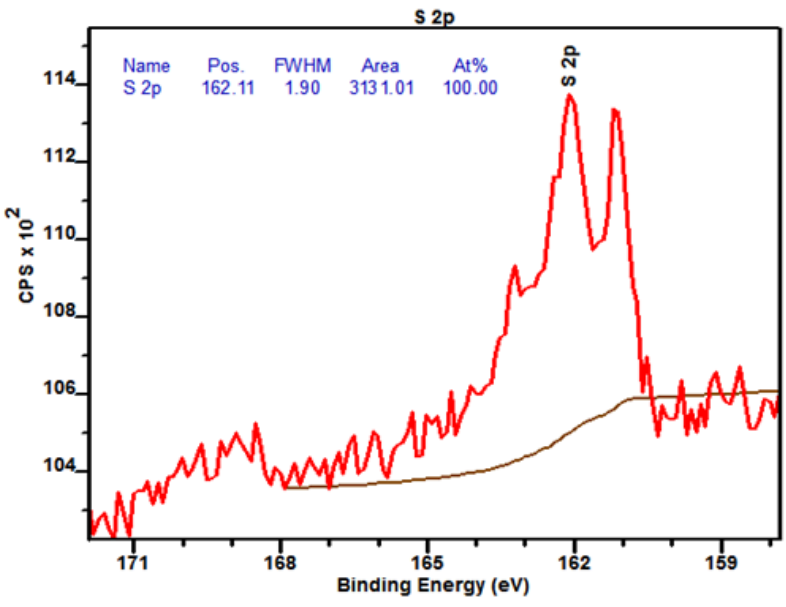

D)

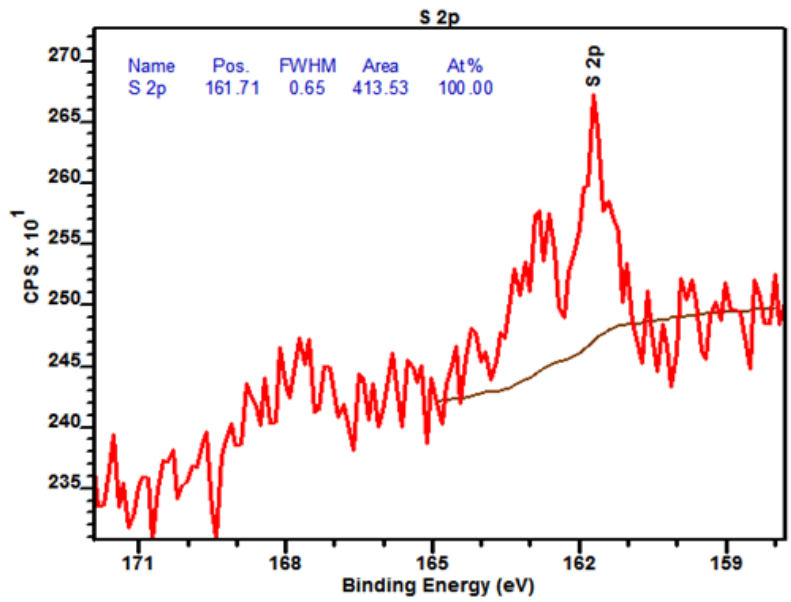

Figure S8. S 2p X-ray photoelectron spectra of A) polygalactose, B) polyglucose, C) $\operatorname{poly}(N$-acetylglucosamine $)$, and $\mathbf{D})$ tethered mannose monomer. 

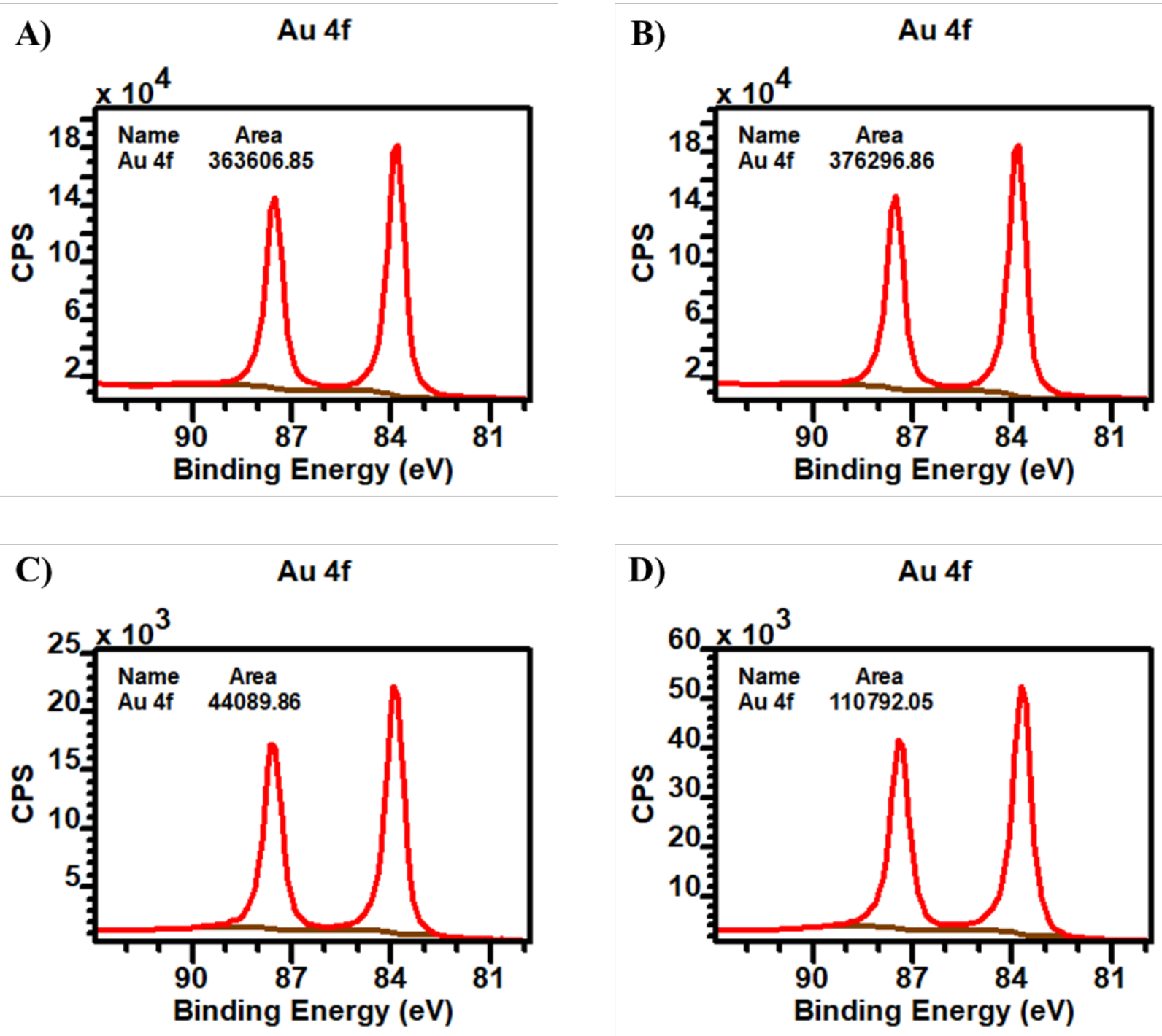

Figure S9. Au 4f X-ray photoelectron spectra of A) polymannose, B) polyglucose,

C) polygalactose, and D) poly( $N$-acetylglucosamine $)$.

The density of glycopolymer molecules can be estimated from the X-ray photoelectron spectroscopy data. The raw areas of the integrated signals are scaled by the relative sensitivity factor of the orbital, 6.25 for $\mathrm{Au} 4 \mathrm{f}$ and 0.668 for $\mathrm{S} 2 \mathrm{p}$. Taking the ratio of these corrected areas provides the ratio of gold to sulfur atoms detected by the instrument. Gold atoms per sulfur atoms are $15 \pm 4$ by standard deviation (SD) for polymannose, $13 \pm 3$ (SD) for polyglucose, $33 \pm$ 
61 (SD) for polygalactose, and $17 \pm 5$ (SD) for poly( $N$-acetylglucosamine). While X-ray photoelectron spectroscopy is a surface-sensitive technique, it is still sensitive to gold atoms beneath the outermost surface layer; thus, the ratio of gold atoms at the molecular interface to sulfur atoms is typically smaller than the measured ratio of total gold to sulfur atoms.

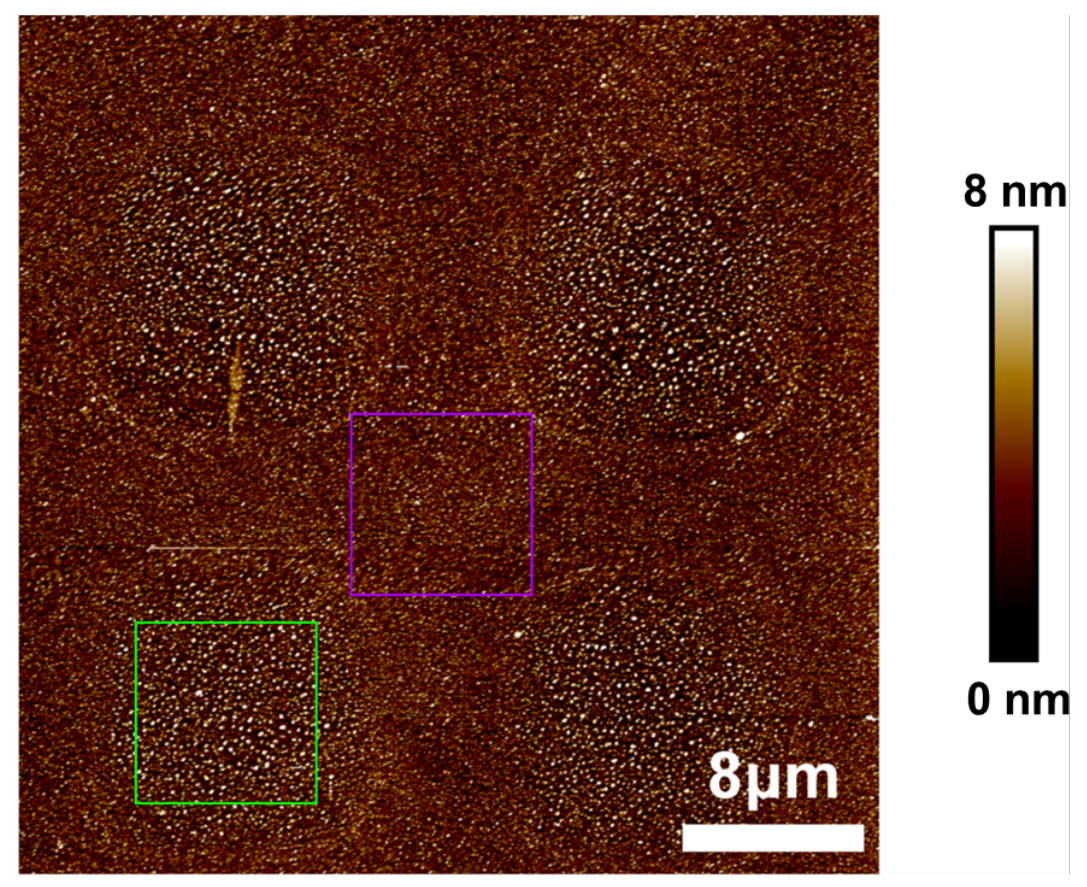

Figure S10. Atomic force micrograph of polymannose pattern on gold surface. The region outlined with the green box corresponds to a polymannose functionalized area and has a rootmean-square surface roughness of $4.4 \mathrm{~nm}$. The region outlined with the purple box corresponds to a bare gold area and has a root-mean-square surface roughness of $4.2 \mathrm{~nm}$. 


\section{MATERIALS AND METHODS}

\section{Materials}

D-Glucose (ACROS, ACS grade), D-galactose (Fisher, off white to white powder), D-mannose (Amresco, high purity grade), D-glucosamine hydrochloride (ACROS, 98+\%), acetic anhydride (Fisher, ACS grade), boron trifluoride etherate (ACROS, 48\%), trimethylsilyl trifluoromethanesulfonate (ACROS, 99\%), trifluoromethanesulfonic acid (ACROS, 99\%), hydrochloric acid (Fisher, ACS grade), phenylmagnesium bromide in 2-methyltetrahydrofuran (Strem, Chemicals, $2.9 \mathrm{M}$ ), carbon disulfide (Fisher, ACS grade), sodium acetate trihydrate (Fisher, ACS grade), 4-dimethylaminopyridine (DMAP) (Alfa Aesar, 99\%), triethylamine (Alfa Aesar, 99\%), sodium bicarbonate (Fisher, USP/FCC), sodium methoxide in methanol (ACROS, 5.4 M), iodine (ACROS, resublimed), methanol (EMD DriSolv, 99.8\%), ethanol (Decon Labs, 200 proof), diethyl ether (Macron Chemicals, ACS grade), tetrahydrofuran (Fisher, HPLC), dichloroethane (ACROS, ACS grade), dimethyl sulfoxide (Fisher, ACS grade), ethyl acetate (Fisher, ACS grade), hexanes (Fisher, ACS grade), DOWEX 50WX8 ion-exchange resin (ACROS, 200-400 mesh) 2-(2-[2-(11-mercaptoundecyloxy)-ethoxy]-ethoxy)-ethoxy-acetic acid (Toronto Research Chemicals), 2-(2-[2-(11-mercaptoundecyloxy)-ethoxy]-ethoxy)-ethanol (Toronto Research Chemicals), 4-aminophenyl- $\alpha$-D-mannopyranoside (LC scientific, 98\%), trifluoroacetic acid (Sigma-Aldrich, 99\%), $N$-hydroxysuccinimide (Aldrich, 98\%), 1ethyl-3-(3-dimethylaminopropyl) carbodiimide (Sigma Aldrich, commercial grade), ethanolamine (Sigma, 98\%), and phosphate-buffered saline (Fisher) were used as received. 4,4'-Azobis(4-cyanovaleric acid) (ACVA) (Pfaltz \& Bauer, 98\%) was recrystallized from methanol. 
Hydroxyethyl acrylate (HEA) (Sigma-Aldrich, 96\%), dichloromethane (Fisher, ACS grade), pyridine (JT Baker, ACS grade), and water (Fisher, HPLC) were distilled for polymer synthesis procedures. Water for surface assembly and bacterial experiments was deionized $(18.2 \mathrm{M} \Omega \mathrm{cm})$ using a Milli-Q system (Millipore, Billerica, Massachusetts, USA).

\section{Synthetic details}

Dialysis was conducted using a Spectra/Por ${ }^{\circledR}$ dialysis membrane (1000 Da) in DI water. Lyophilization was conducted on a Labconco FreeZone 4.5 freeze dryer. Gel permeation chromatography (GPC) was conducted on a Jasco system equipped with a UV detector, a refractive index detector, and four Waters ultrahydrogel columns $(100-5 \mathrm{~K}, 1 \mathrm{~K}-80 \mathrm{~K}, 10 \mathrm{~K}-400 \mathrm{~K}$, $2 \mathrm{~K}-4 \mathrm{M}, 500-10 \mathrm{M}$ ) using $10 \mathrm{mM} \mathrm{PBS}$ with $0.3 \mathrm{M} \mathrm{NaCl}$ at $\mathrm{pH} 6.6$ as an eluent at $30^{\circ} \mathrm{C}$ and a flow rate of $1.0 \mathrm{~mL} / \mathrm{min}$ calibrated using pullulan standards.

\section{Co-culture competition experiment}

Cultures of both S. oneidensis MR-1 WT p519nGFP and S. oneidensis $\Delta m s h \mathrm{~A}-\mathrm{D}$ were grown and resuspended in PBS as described in the main text. Due to differing growth rates the optical density of the initial cell cultures was not grown to 0.9 . Instead, both strains were incubated for a fixed time of $2.5 \mathrm{~h}$. The two strains were mixed together and diluted before adding to the surfaces of interest. The volumes of each strain added were adjusted based on optical density to have the same number of cells of each type added to the surfaces. The samples were incubated at $32{ }^{\circ} \mathrm{C}$ for $18 \mathrm{~h}$ with shaking at $200 \mathrm{rpm}$. The surfaces were then mounted on a glass coverslip and imaged.

An upright ZEISS LSM-800 confocal microscope (Carl Zeiss Microscopy, LLC, White Plains, New York, USA) was used for optical imaging of cells. Green fluorescent protein was excited at 
$488 \mathrm{~nm}$. Brightfield was imaged with confocal reflection at $640 \mathrm{~nm}$ and detected at the same wavelength by photomultiplier tube.

Unpaired t-test were used for statistical comparison of the samples.

\section{Ellipsometry}

Ellipsometry was measured with a Gaertner LSE Stokes Ellipsometer (Gaertner Scientific Corporation, Skokie, Illinois, USA). A $632.8 \mathrm{~nm}$ HeNe laser was used at a $70^{\circ}$ incidence angle. Each glycopolymer was self-assembled on 100-nm-thick Au substrates produced by electronbeam evaporation.

\section{Electrochemical desorption}

A BASi Epsilon potentiostat (Bioanalytical Systems Inc., West Lafayette, Indiana, USA) was used to measure the current during electrochemical desorption. The voltage was swept from 0 to $-1200 \mathrm{mV}$ with a $\mathrm{Ag} / \mathrm{AgCl}$ reference electrode at a scan rate of $50 \mathrm{mV} / \mathrm{s}$. Pt was used as the counter electrode in a $0.5 \mathrm{M} \mathrm{KOH}$ electrolyte solution. A 1/8-inch inner diameter O-ring was used to define the effective surface area of the analyte.

\section{Contact angle}

A FTA1000 Drop Shape Instrument (First Ten Angstroms, Inc., Portsmouth, Virginia, USA) was used to record images of water droplets on the surfaces and calculate contact angles with the associated software. Water was applied the surfaces by a syringe. It was pumped in and out of the syringe repeatedly to determine receding and advancing contact angles, respectively. 


\section{Polarization modulation-infrared reflection-absorption spectroscopy}

A Nicolet 8700 FT-IR spectrometer, with Hinds Instruments PEM-100 photoelastic modulator, and MCTA detector (Thermo Scientific, Wilmington, Delaware, USA) was used for PM-IRRAS measurements. The sample chamber was purged with dry nitrogen gas before recording. Spectra were acquired over the range of $1000-3500 \mathrm{~cm}^{-1}$. The PEM wavelength was set to $3100 \mathrm{~cm}^{-1}$. Measurements were averaged over 2048 scans with a resolution of $2 \mathrm{~cm}^{-1}$.

\section{Nuclear magnetic resonance}

${ }^{1} \mathrm{H}$ NMR spectra were acquired with a Bruker AV-300 spectrometer. Glycopolymer samples were dissolved in $\mathrm{D}_{2} \mathrm{O}$ and scanned in a $300 \mathrm{MHz}$ magnetic field.

\section{X-ray photoelectron spectroscopy}

An AXIS Ultra DLD X-ray photoelectron spectrometer (Kratos Analytical Inc., Chestnut Ridge,

New York, USA) was used for elemental surface analysis. This spectrometer uses a

monochromatic $\mathrm{Al} \mathrm{K} \mathrm{K}_{\alpha} \mathrm{X}$-ray source with a $200 \mu \mathrm{m}$ circular spot size and ultrahigh vacuum $\left(10^{-9}\right.$ Torr). Spectra were acquired at a pass energy of $160 \mathrm{eV}$ for survey spectra and $20 \mathrm{eV}$ for

high-resolution spectra of C 1s, N 1s, O 1s, S 2p, and Au 4f regions using a $250 \mathrm{~ms}$ dwell time. The emission current was $10 \mathrm{~mA}$ and $20 \mathrm{~mA}$ for survey and high-resolution spectra, respectively. The applied potential was $15 \mathrm{kV}$ for both scan types. Three scans were performed for survey and Au spectra, 10 scans for N, C, and O spectra, and 15 scans for S spectra.

\section{Patterning polymannose by microcontact printing}

Patterned PDMS stamps were formed on silicon masters with $10 \mu \mathrm{m}$ square features. The stamps were soaked in hexane for 1 hour then removed. A PDMS stamp was submerged in a solution of 
$15 \mathrm{mg}$ of poly(mannose acrylate), $48 \mu \mathrm{L}$ of ethanolamine, and $8 \mathrm{~mL}$ of water and left overnight. The stamp was removed from solution, rinsed with water and ethanol, dried, and printed on a flame-annealed $100 \mathrm{~nm}$ thick gold surface adhered to a silicon substrate by a titanium adhesion

layer. A $38 \mathrm{~g}$ weight was placed on the stamp and allowed to sit for $2 \mathrm{~h}$. The stamp was removed from the gold sample.

\section{Atomic force microscopy}

The microcontact printed sample was scanned with a Dimension Icon atomic force microscope (Bruker, Billerica, Massachusetts, USA) using ScanAsyst-Air probes (Bruker). 